Pacific Journal of Mathematics

INDICATOR FUNCTIONS WITH LARGE FOURIER 


\section{INDICATOR FUNCTIONS WITH \\ LARGE FOURIER TRANSFORMS}

\section{Gavin Brown, Irving Glicksberg and Edwin HewitT}

We consider the question of when the function

$$
t \mapsto t \hat{1}_{F}(t)
$$

is bounded, where $1_{F}$ is the indicator function of a compact set $F$ in $\mathbf{R}$ and " " denotes the Fourier transform.

We are concerned in this note with a question of P. R. Masani about the rate of decrease of certain Fourier transforms on the real line $\mathbf{R}$. Throughout, all unexplained notation is as in [1]. For $f \in \mathfrak{L}_{1}(\mathbf{R})$, we write

$$
\hat{f}(t)=(2 \pi)^{-1 / 2} \int_{-\infty}^{\infty} f(x) \exp (\text { itx }) d x \quad(t \in \mathbf{R}) .
$$

(It is convenient to use $\exp ($ itx $)$ in the integral in (1) in place of the equally common $\exp (-i t x)$.)

Masani has asked whether or not there exist compact subsets $F$ of $\mathbf{R}$ with Lebesgue measure $\lambda(F)>0$ such that the function

$$
t \mapsto t \hat{1}_{F}(t)
$$

is unbounded. By the Cantor-Bendixson theorem, we may suppose that $F$ is perfect. For a bounded closed interval $[a, b] \subset \mathbf{R}$, the function $t \hat{1}_{[a, b]}(t)$ is

$$
-i(2 \pi)^{-1 / 2}(\exp (i b t)-\exp (\text { iat }))
$$

which is trivially bounded. For $a=\inf F$ and $b=\sup F$, write $U=$ $[a, b] \backslash F$ and get

$$
t \hat{\mathrm{1}}_{F}(t)+t \hat{\mathrm{1}}_{U}(t)=-i(2 \pi)^{-1 / 2}(\exp (i b t)-\exp (\text { iat })),
$$

so that the function (2) is bounded if and only if the function

$$
t \mapsto t \hat{1}_{U}(t)=h_{U}(t)
$$

is bounded. Thus Masani's problem is equivalent to the problem of finding bounded open subsets $U$ of $\mathbf{R}$ whose complements contain no isolated points and for which the function $h_{U}$ is unbounded.

We note a simple case in which $h_{U}$ is bounded. Suppose that

$$
\lambda([\inf U, \sup U] \backslash U)=0,
$$


as happens for example if $U$ is the union of the complementary intervals in $[0,1]$ of Cantor's ternary set. Then (4) and (6) give

$$
t \cdot 0+t \hat{1}_{U}(t)=-i(2 \pi)^{-1 / 2}(\exp (i(\sup U) t)-\exp (i(\inf U) t)) .
$$

The same holds if $U$ is the union of a finite family of open sets for each of which (6) holds.

We have no complete classification of the open subsets $U$ of $\mathbf{R}$ for which the function $h_{U}$ is bounded. However, there is one special case where the answer is clear, as a consequence of a theorem of L. H. Loomis [3].

Given a closed subset $F$ of $\mathbf{R}$, let $P(F)$ be the set of all condensation points $x$ of $F$ (every neighborhood of $x$ contains an uncountable subset of $F)$. As is well known, $P(F)$ is perfect or void and $F \backslash P(F)$ is countable.

Theorem A. Suppose that the bounded open subset $U$ of $\mathbf{R}$ is the union of a countably infinite family of non-abutting open intervals \{]$a_{j}, b_{j}[\}_{j=1}^{\infty}$ and that the boundary $\partial U=U^{-} \backslash U$ has an accumulation point outside of the perfect set $P(\partial U) .{ }^{1}$ Then the function $h_{U}$ is unbounded.

Proof. For convenience we will use $\mathfrak{S}$, the usual space of rapidly decreasing complex-valued $C^{\infty}$ functions on $\mathbf{R}$. The Fourier transformation (1) maps $\subseteq$ onto the corresponding space of functions on the dual line. The identity

$$
\left(g^{\prime}\right)^{\hat{(}}(t)=-i t \hat{g}(t) \quad(g \in \Im)
$$

is standard.

We now assume that $h_{U}$ is bounded. We will ultimately obtain a contradiction. For all real-valued $g \in \mathfrak{S},(8)$ and Parseval's identity give

$$
\begin{aligned}
i h_{U} * \hat{g}(0) & =i(2 \pi)^{-1 / 2} \int_{-\infty}^{\infty} t \hat{1}_{U}(t) \hat{g}(-t) d t \\
= & (2 \pi)^{-1 / 2} \int_{-\infty}^{\infty} \hat{1}_{U}(t) \overline{(-i t \hat{g}(t))} d t \\
= & (2 \pi)^{-1 / 2} \int_{-\infty}^{\infty} \hat{1}_{U}(t) \overline{\left(g^{\prime}\right)^{\hat{(}(t)}} d t \\
= & (2 \pi)^{-1 / 2} \int_{-\infty}^{\infty} 1_{U}(x) g^{\prime}(x) d x \\
= & (2 \pi)^{-1 / 2} \sum_{j=1}^{\infty} \int_{a_{J}}^{b_{J}} g^{\prime}(x) d x=(2 \pi)^{-1 / 2} \sum_{j=1}^{\infty}\left(g\left(b_{j}\right)-g\left(a_{j}\right)\right) .
\end{aligned}
$$

\footnotetext{
${ }^{1}$ Note that $P(\partial U)$ is void if and only if $\partial U$ is countable. In this case any accumulation point of $\partial U$ will serve our purpose.
} 
Let $f$ be any real-valued function in $\subseteq$ and let $s$ be a fixed real number. Replace $g$ in (9) by the function

$$
t \rightarrow \hat{f} * \hat{g}(s+t)
$$

The identity (9) becomes

(10) $i h_{U} * \hat{f} * \hat{g}(s)$

$$
=(2 \pi)^{-1 / 2} \sum_{j=1}^{\infty}\left[f\left(b_{j}\right) g\left(b_{j}\right) \exp \left(i b_{j} s\right)-f\left(a_{J}\right) g\left(a_{J}\right) \exp \left(i a_{j} s\right)\right] .
$$

Now consider a point of accumulation $x_{0}$ of $\partial U$ that does not lie in $P(\partial U)$. There is a real valued function $f$ in $\subseteq$ such that $f\left(x_{0}\right)=1$ and $f$ vanishes in an open neighborhood $V$ of the set $P(\partial U)$. We choose and fix such a function $f$. Let $g$ be a real-valued function in $\subseteq$ that vanishes on $\partial U \backslash V$. For such a function $g$, the function $f g$ vanishes at all of the points $a_{j}$ and $b_{j}$, as a moment's thought shows. Thus the identity (10) shows that

$$
h_{U} * \hat{f} * \hat{g}=0 \text {. }
$$

For each $x$ not in $\partial U \backslash V$, we can define the real-valued function $g$ in $\subseteq$ so that $g(x)=1$ and so that $g$ vanishes on $\partial U \backslash V$. Therefore the spectrum of the function $h_{U} * \hat{f}$ is contained in the countable closed set $\partial U \backslash V$, which is contained in $\partial U \backslash P(\partial U)$. Loomis ([3], Theorem 4) has shown that a bounded measurable function on a locally compact Abelian group $G$ whose spectrum is compact and contains no nonvoid perfect subset is almost periodic. (For the present case, $G=\mathbf{R}$, these are exactly the functions in $\mathfrak{R}_{\infty}(\mathbf{R})$ with bounded countable spectrum.) Therefore the function $h_{U} * \hat{f}$ is continuous and almost periodic for all functions $f$ of the form described above.

Now let

$$
t \rightarrow \sum_{k=1}^{n} \mu_{k} \exp \left(i c_{k} t\right)=p_{f}(t)
$$

be a trigonometric polynomial on $\mathbf{R}$ such that

$$
\left\|h_{U} * \hat{f}-p_{f}\right\|_{\infty}<\frac{1}{4} .
$$

Computing a convolution at 0 , we use (11) and (10) to infer that

$$
\begin{aligned}
\frac{1}{4}\|g\|_{1} & \geq\left|\left(h_{U} * \hat{f}-p_{f}\right) * \hat{g}(0)\right| \\
& =\left|\sum_{j=1}^{\infty}\left[f\left(b_{j}\right) g\left(b_{j}\right)-f\left(a_{j}\right) g\left(a_{j}\right)\right]-\sum_{k=1}^{n} \mu_{k} g\left(c_{k}\right)\right| .
\end{aligned}
$$


Since $f\left(x_{0}\right)=1$, there is an open neighborhood $W$ of $x_{0}$ with compact closure such that $|f(x)| \geq \frac{3}{4}$ for all $x \in W^{-}$. Plainly $W^{-}$and $P(\partial U)$ are disjoint. Since $W \cap(\partial U)$ is (countably) infinite and disjoint from $P(\partial U)$, it contains a point $x_{1}$ of $\partial U$ that is isolated in $\partial U$ and is different from all of the points $c_{1}, c_{2}, \ldots, c_{n}$. Note that $x_{1}$ cannot be $x_{0}$ and that the only possible isolated points of $\partial U$ are endpoints $a_{j}$ and $b_{j}$ of the component intervals of $U$.

Suppose that we have a real-valued function $g$ in $\subseteq$ such that $g\left(x_{1}\right)=1, g$ vanishes in a neighborhood of the compact set $\left(\partial U \backslash\left\{x_{1}\right\}\right) \cup$ $\left\{c_{1}, c_{2}, \ldots, c_{n}\right\}$, and $\|\hat{g}\|_{1}=1$. Put this $g$ into formula (12). Since $f$ vanishes on $P(\partial U)$ and $g$ vanishes on $\partial U$ except at $x_{1}$, the only surviving term in the second line of (12) is $\pm f\left(x_{1}\right) g\left(x_{1}\right)$. Since $\left|f\left(x_{1}\right)\right| \geq \frac{3}{4}$ by construction, (12) yields

$$
\frac{1}{4} \geq\left|f\left(x_{1}\right) g\left(x_{1}\right)\right| \geq \frac{3}{4}\left|g\left(x_{1}\right)\right|=\frac{3}{4},
$$

a contradiction. Therefore the function $h_{U}$ is unbounded.

To finish the proof, we need only to find a function with the properties ascribed to $g$ in the preceding paragraph. This is standard save for the requirement that $g$ be in $\subseteq$. Imitating the standard construction, we suppose first that $x_{1}=0$. Let $\delta$ be any positive real number, and take $\psi$ to be an even nonnegative $C^{\infty}$ function with support $\left[-\frac{1}{2} \delta, \frac{1}{2} \delta\right]$ for which

$$
(2 \pi)^{-1 / 2} \int_{-\infty}^{\infty} \psi^{2}(x) d x=1
$$

Define $g$ as the convolution $\psi * \psi$. Plainly $g$ is in $\subseteq$ and has support $[-\delta, \delta]$. Since $\psi$ is real-valued, we have

$$
g(0)=(2 \pi)^{-1 / 2} \int_{-\infty}^{\infty} \psi(x) \psi(-x) d x=1
$$

and

$$
\begin{aligned}
\|\hat{g}\|_{1} & =(2 \pi)^{-1 / 2} \int_{-\infty}^{\infty} \hat{g}(t) d t=(2 \pi)^{-1 / 2} \int_{-\infty}^{\infty} \hat{\psi}(t)^{2} d t \\
& =(2 \pi)^{-1 / 2} \int_{-\infty}^{\infty} \psi^{2}(x) d x=1 .
\end{aligned}
$$

For $x_{1} \neq 0$, use the translated function $x \rightarrow g\left(-x_{1}+x\right)$, whose support is $\left[x_{1}-\delta, x_{1}+\delta\right]$ and whose Fourier transform at $t$ is $\exp \left(i x_{1} t\right) \hat{g}(t)$.

REMARKS. Let $\left(\gamma_{j}\right)_{j=1}^{\infty}$ be any bounded sequence of complex numbers such that $\left(\left|\gamma_{j}\right|\right)_{j=1}^{\infty}$ is bounded away from zero. Consider the function

$$
\varphi=\sum_{j=1}^{\infty} \gamma_{j} 1_{] a_{j}, b_{j}[},
$$


where the open set $\left.U=\cup_{j=1}^{\infty}\right] a_{j}, b_{j}$ [ satisfies the hypotheses of Theorem A. The proof of Theorem A can be repeated with an obvious modification in (11) to prove that the function

$$
t \rightarrow t \hat{\varphi}(t)
$$

is unbounded. If $f$ is a continuous function on $\mathbf{R}$ such that $f^{\prime}$ exists except possibly at a countable set of points and if both $f$ and $f^{\prime}$ are in $\mathfrak{I}_{1}(\mathbf{R})$, then $f$ is absolutely continuous and

$$
\left(f^{\prime}\right)^{\hat{n}}(t)=-i \hat{f}(t)
$$

for all $t \in \mathbf{R}$. Thus the function

$$
t \rightarrow t \hat{f}(t)
$$

is not only bounded but is $o(1)$. Adding to such $f$ any function $\varphi$ of the form (14), we get more functions $g$ in $\mathfrak{L}_{1}(\mathbf{R})$ for which the function

$$
t \rightarrow t \hat{g}(t)
$$

is unbounded.

EXAMPLE A. Let \{]$a_{j}, b_{j}[\}_{j=1}^{\infty}$ be a countably infinite family of nonvoid, non-abutting open intervals in $\mathbf{R}$ and as above write $U$ for the set $\left.\cup_{j=1}^{\infty}\right] a_{j}, b_{j}$ [. Suppose that $U$ is bounded. It is easy to see that $\partial U$ is the closure of the countable set $H=\left\{a_{1}, a_{2}, \ldots, a_{n}, \ldots\right\} \cup\left\{b_{1}, b_{2}, \ldots, b_{n}, \ldots\right\}$. If $H^{-}$is countable, then the open set $U$ satisfies the hypotheses of Theorem A, since the perfect set $P(\partial U)=P\left(H^{-}\right)$is void. A continuum of such open sets exist and can be constructed ad libitum. Thus open sets $U$ for which $h_{U}$ is unbounded exist in profusion.

EXAMPLE B. We now present a construction that is roughly the antithesis of Example $\mathrm{A}$, in that the set $H$ consists solely of isolated points, while the set $P\left(\mathrm{H}^{-}\right)$is equal to $\mathrm{H}^{-} \backslash H$ and is homeomorphic to Cantor's ternary set. At the same time the function $h_{U}$ is unbounded for this set $U$. Thus we will show that the hypotheses of Theorem A are not necessary in order for the function $h_{U}$ to be unbounded.

For every positive integer $n$, let $E_{n}$ be the set of all sequences $\varepsilon=\left(\varepsilon_{1}, \varepsilon_{2}, \ldots, \varepsilon_{n}\right)$ where each entry $\varepsilon_{j}$ is either 1 or -1 . Let $C_{n}$ be the subset of $E_{n}$ consisting of all $\varepsilon$ with $\varepsilon_{1}=1$. For each $\varepsilon$ in $E_{n}$, let $I(n, \varepsilon)$ be the open interval

$$
] \sum_{j=1}^{n} \varepsilon_{j} 4^{-j}-\frac{1}{2} 4^{-n-1}, \sum_{j=1}^{n} \varepsilon_{j} 4^{-j}+\frac{1}{2} 4^{-n-1}[.
$$

Let $U$ be the union of all of the intervals $I(n, \varepsilon)$ as $\varepsilon$ runs through all of the $2^{n}$ elements of $E_{n}$ and $n$ runs through the set of all positive integers. 
We find that

$$
I(n, \varepsilon) \cap I\left(n^{\prime}, \varepsilon^{\prime}\right)=\varnothing
$$

unless $n=n^{\prime}$ and $\varepsilon=\varepsilon^{\prime}$. As in Example A, write $H$ for the set of all endpoints of all of the intervals $I(n, \varepsilon)$. Let $D$ be the set of all numbers of the form

$$
\sum_{j=1}^{\infty} \beta_{j} 4^{-j}
$$

where each $\beta$, is either 1 or -1 . We find that

$$
D=H^{-} \backslash H=\partial U \text {. }
$$

The details of proving (16) and (17) are simple enough but are also somewhat tedious, and we omit them. Note that

$$
\sup U=\frac{1}{3}, \quad \inf U=-\frac{1}{3}, \text { and } \lambda(U)=\frac{1}{4} .
$$

We now compute the function $h_{U}$.

Given an interval $] c-\gamma, c+\gamma[(c \in \mathbf{R}, \gamma>0)$, we have

$$
\exp (i(c+\gamma) t)-\exp (i(c-\gamma) t)=2 i \sin (\gamma t) \exp (i c t) \text {. }
$$

For every positive integer $n$, (3) and (19) show that

$$
\begin{aligned}
& \sum_{\varepsilon \in E_{n}} t \hat{1}_{I(n, \boldsymbol{e})}(t)=\sum_{\varepsilon \in E_{n}} 2 \sin \left(\frac{1}{2} 4^{-n-1} t\right) \exp \left(i\left(\sum_{j=1}^{n} \varepsilon_{j} 4^{-j}\right) t\right) \\
& =\sum_{\varepsilon \in C_{n}} 2 \sin \left(\frac{1}{2} 4^{-n-1} t\right)\left[\exp \left(i\left(\sum_{j=1}^{n} \varepsilon_{j} 4^{-j}\right) t\right)\right. \\
& \left.+\exp \left(i\left(-\sum_{j=1}^{n} \varepsilon_{j} 4^{-j}\right) t\right)\right] \\
& =2 \sin \left(\frac{1}{2} 4^{-n-1} t\right) \prod_{r=1}^{n}\left[\exp \left(i 4^{-r} t\right)+\exp \left(-i 4^{-r} t\right)\right] \\
& =2^{n+1} \sin \left(\frac{1}{2} 4^{-n-1} t\right) \prod_{r=1}^{n} \cos \left(4^{-r} t\right) .
\end{aligned}
$$

Add (20) over all positive integers $n$ to obtain

$$
h_{U}(t)=\sum_{n=1}^{\infty} 2^{n+1} \sin \left(\frac{1}{2} 4^{-n-1} t\right) \prod_{r=1}^{n} \cos \left(4^{-r} t\right) .
$$

For a given positive integer $p$, let us compute (21) for $t=2 \pi 4^{p}$. For $n=1,2, \ldots, p-1$, we have

$$
\sin \left(\frac{1}{2} 4^{-n-1} 2 \pi 4^{p}\right)=\sin \left(\pi 4^{p-n-1}\right)=0 .
$$


For $n=p$, we have

$$
\sin \left(\frac{1}{2} 4^{-p-1} 2 \pi 4^{p}\right)=\sin \left(\frac{1}{2} \pi\right)=2^{-1 / 2} .
$$

Also for $n=p$, we have

$$
\prod_{r=1}^{p} \cos \left(4^{-r} 2 \pi 4^{p}\right)=\prod_{r=1}^{p} \cos \left(2 \pi 4^{p-r}\right)=1 .
$$

For $n \geq p+1$, we have

$$
\prod_{r=1}^{n} \cos \left(2 \pi 4^{p-r}\right)=0
$$

since

$$
\cos \left(2 \pi 4^{p-p-1}\right)=\cos \left(\frac{1}{2} \pi\right)=0 .
$$

Combining (21)-(25), we see that

$$
h_{U}\left(2 \pi 4^{p}\right)=2^{p+1 / 2},
$$

so that $h_{U}(t)$ is unbounded.

It is of some interest to examine the rate of growth of the function $h_{U}(t)$ for $U$ 's as in Theorem A.

EXAMPLE C. Let $\varphi$ be any continuous nondecreasing function on $\left[1, \infty\left[\right.\right.$ such that $\lim _{t \rightarrow \infty} \varphi(t)=\infty$. We can find a bounded open set $U$ such that $h_{U}(t)$ is unbounded and

$$
h_{U}(t)=O(\varphi(|t|)) .
$$

To find such a set $U$, let $\psi=\psi(u)$ be the function defined on $[\varphi(1), \infty$ [ such that: if $\varphi$ assumes the value $u$ at exactly one point $t$, then $\psi(u)=t$; if $\varphi$ assumes the value $u$ exactly in an interval $[a, b]$ with $a<b$, then $\psi(u)=b$. That is, $\psi$ is as close to the inverse function of $\varphi$ as one can get. It is plain that $\lim _{u \rightarrow \infty} \psi(u)=\infty$ and that $\psi$ is strictly increasing. that

It is easy to construct an infinite series $\sum_{n=1}^{\infty} r_{n}$ of positive terms such

$$
\sum_{n=N+1}^{\infty} r_{n}=\frac{1}{\psi(N+1)}
$$

for all positive integers $N$. Let \{]$a_{n}, b_{n}[\}_{n=1}^{\infty}$ be a set of open intervals with the following properties for all $n$ :

$$
a_{n}<b_{n} ; \quad b_{n}-a_{n}=r_{n} ; \quad b_{n+1}<a_{n} ;
$$

and

$$
\lim _{n \rightarrow \infty} a_{n}=0 \text {. }
$$


It is plain that $P(\partial U)=\varnothing$, and so by Theorem $\mathrm{A}$ the function $h_{U}(t)$ is unbounded. For every positive integer $N$, we have

$$
\begin{aligned}
\left|h_{U}(t)\right| \leq & \mid \sum_{n=1}^{N}\left(\exp \left(i b_{n} t\right)-\exp \left(i a_{n} t\right) \mid\right. \\
& +\mid \sum_{n=N+1}^{\infty}\left(\exp \left(i b_{n} t\right)-\exp \left(i a_{n} t\right) \mid\right. \\
\leq & 2 N+|t| \sum_{n=N+1}^{\infty}\left(b_{n}-a_{n}\right)=2 N+|t| \frac{1}{\psi(N+1)} .
\end{aligned}
$$

Given a real number $t$ of absolute value at least 1 , let $N$ be the integer such that

$$
N \leq \varphi(|t|)<N+1
$$

This gives us

$$
\psi(N) \leq \psi(\varphi(|t|))<\psi(N+1) .
$$

By our definition of $\psi$, we have

$$
\begin{gathered}
|t| \leq \psi(\varphi(|t|)), \\
\left|h_{U}(t)\right| \leq 2 \varphi(|t|)+\psi(N+1) \frac{1}{\psi(N+1)}=O(\varphi(|t|)) .
\end{gathered}
$$

Thus the function $h_{U}(t)$ can go to infinity arbitrarily slowly.

Finally we compute the exact rate of growth of the function $h_{U}(t)$ for the open set $U$ of Example B. The equality (26) shows that

$$
\left|h_{U}(t)\right| \geq C t^{1 / 2}
$$

for arbitrarily large positive values of $t$. On the other hand, consider all of the intervals $I(n, \varepsilon)$ for $n \leq N, N$ being an arbitrary positive integer. There are exactly $2^{N+1}-1$ such intervals. The sum of the measures of all of the intervals $I(n, \varepsilon)$ for $n \geq N+1$ is $2^{-N-2}$. Accordingly, (28) shows that

$$
\left|h_{U}(t)\right| \leq 2\left(2^{N+1}-1\right)+|t| 2^{-N-2} .
$$

For a given $t$ of absolute value at least 4 , define $N$ by

$$
2^{2 N+2} \leq|t|<2^{2 N+3} \text {. }
$$

From (30) we get

$$
\left|h_{U}(t)\right| \leq 2|t|^{1 / 2}+2^{-1 / 2}|t|^{1 / 2}
$$


so that

$$
\left|h_{U}(t)\right|=O\left(|t|^{1 / 2}\right) .
$$

The estimates (29) and (31) show that $\left|h_{U}(t)\right|=O\left(|t|^{\alpha}\right)$ for $\alpha=\frac{1}{2}$ but for no smaller exponent $\alpha$.

We are indebted to Professor Masani for the following remarks on the origin of his problem.

Question. Let $\mathfrak{X}$ be a complex Banach space, and let $\{(U(t): t \in \mathbf{R}\}$ be a strongly continuous group of linear isometries of $\mathfrak{X}$ onto $\mathfrak{X}$ with infinitesimal generator $A$. For what bounded Borel subsets $S$ of $\mathbf{R}$ is it the case that

$$
\text { Range } \int_{S} U(t) d t \subset \text { Dom } A \text { ? }
$$

This question arises naturally in the theory of $\mathfrak{X}$-valued stationary measures over R. See [4], page 303, Theorem 3.6. The inclusion (32) holds provided that $S$ is a closed interval. This is proved in [2], §10.3, page 307. Thus (32) holds if $S$ is a union of finitely many closed intervals.

Now suppose that $\mathfrak{X}$ is a Hilbert space. The problem of the inclusion (32) reduces to the problem of Masani stated in the second paragraph of this note. To see this, write

$$
U(t)=\int_{\mathbf{R}} \exp (i t x) d(E(x)), \text { so that } A=\int_{\mathbf{R}} i x d(E(x)) .
$$

It is then easy to see that

$$
\int_{S} U(t) d t=\int_{\mathbf{R}} \hat{1}_{S}(x) d(E(x))
$$

and that

$$
A \int_{S} U(t) d t \subset \int_{\mathbf{R}} i x \hat{1}_{S}(x) d(E(x)) .
$$

Now (32) holds if and only if the operator on the left side of (33) is continuous on $\mathfrak{X}$, that is, if and only if the function $x \mapsto x \hat{1}_{S}(x)$ is $E$-essentially bounded on $\mathbf{R}$. It is also easy to see that a bounded Borel set $S$ satisfies (32) for all $U(\cdot)$ if and only if the function $x \mapsto x \hat{1}_{S}(x)$ is bounded on $\mathbf{R}$. Thus finding the bounded Borel sets satisfying (32) yields the problem stated in the second paragraph of this note.

Finally we remark that Masani [4], page 304, Proposition 3.8, has proved a special case of Example A. 


\section{REFERENCES}

1. Edwin Hewitt and Kenneth A. Ross, Abstract harmonic analysis, Vols. I and II, Berlin-Heidelberg-New York, 1979, 1970.

2. Einar Hille and Ralph S. Phillips, Functional Analysis and Semi-groups, 2nd edition, Providence, R. I.: American Mathematical Society, 1957.

3. Lynn H. Loomis, The spectral characterization of a class of almost periodic functions, Ann. of Math., (2) 72 (1960), 362-368.

4. P. R. Masani, An Outline of the Theory of Stationary Measures Over $\mathbf{R}^{q}$, Berlin-Heidelberg-New York: Springer-Verlag, Lecture Notes in Mathematics \#794, 1980, Measure Theory Oberwolfach 1979, Proceedings, pages 295-309.

Received July 7, 1981 and in revised form January 19, 1982. The research of all three authors was supported in part by the National Science Foundation, USA.

UNIVERSITY OF NeW SOUTH Wales

KENSINGTON NSW 2033

AND

UNIVERSITY OF WASHINGTON

SEATtLE, WA 98195 


\section{PACIFIC JOURNAL OF MATHEMATICS}

EDITORS

Donald BABBITT (Managing Editor)

University of California

Los Angeles, CA 90024

Hugo Rossi

University of Utah

Salt Lake City, UT 84112

C. C. Moore and Arthur Ogus

University of California

Berkeley, CA 94720
J. DugunduI

Department of Mathematics

University of Southern California

Los Angeles, CA 90089-1113

R. FinN and H. SAmelson

Stanford University

Stanford, CA 94305

\section{ASSOCIATE EDITORS}
R. ARENS
E. F. BECKENBACH
B. H. NEUMANN
F. WolF
K. YoshidA (1906-1982)

\section{SUPPORTING INSTITUTIONS}

UNIVERSITY OF ARIZONA

UNIVERSITY OF BRITISH COLUMBIA

CALIFORNIA INSTITUTE OF TECHNOLOGY

UNIVERSITY OF CALIFORNIA

MONTANA STATE UNIVERSITY

UNIVERSITY OF NEVADA, RENO

NEW MEXICO STATE UNIVERSITY

OREGON STATE UNIVERSITY
UNIVERSITY OF OREGON

UNIVERSITY OF SOUTHERN CALIFORNIA

STANFORD UNIVERSITY

UNIVERSITY OF HAWAII

UNIVERSITY OF TOKYO

UNIVERSITY OF UTAH

WASHINGTON STATE UNIVERSITY

UNIVERSITY OF WASHINGTON 


\section{Pacific Journal of Mathematics}

Vol. 105, No. 1 September, 1983

Kenneth F. Andersen, On the transformation of Fourier coefficients of

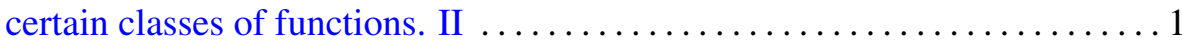

Gavin Brown, Irving Leonard Glicksberg and Edwin Hewitt, Indicator functions with large Fourier transforms $\ldots \ldots \ldots \ldots \ldots \ldots \ldots \ldots \ldots \ldots$

Shih-Sen Chang, Some random fixed point theorems for continuous random

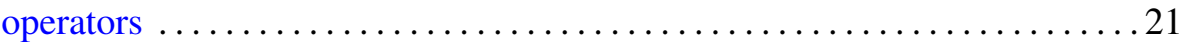

K. C. Chattopadhyay and Olav Njstad, Quasiregular nearness spaces and

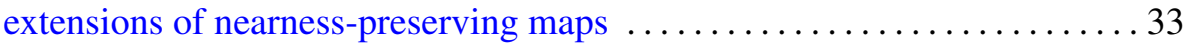

Thomas W. Cusick, The two-dimensional Diophantine approximation

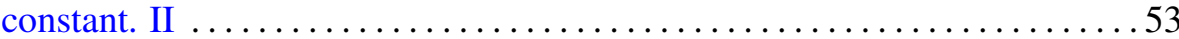

Eric Karel van Douwen and Jan van Mill, Spaces without remote points . . .669 Hector O. Fattorini, Convergence and approximation theorems for

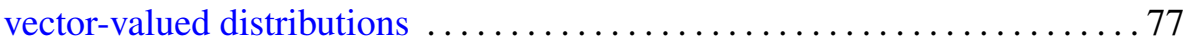

John J. F. Fournier and Louis Pigno, Analytic and arithmetic properties of

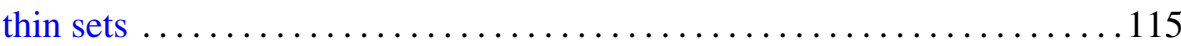

David Goss, On a new type of $L$-function for algebraic curves over finite

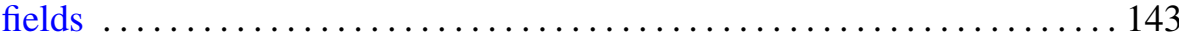

Douglas Austin Hensley, Lattice vertex polytopes with interior lattice

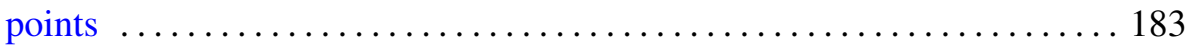

J. K. Kohli, Monotone extensions of mappings and their applications ...... 193

John C. Morgan, II, On equivalent category bases . . . . . . . . . . . . 207

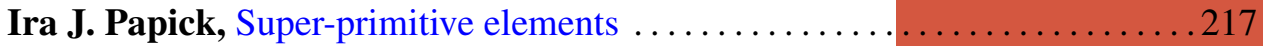

José Luis Rubio de Francia and José Luis Torrea, Vector extensions of

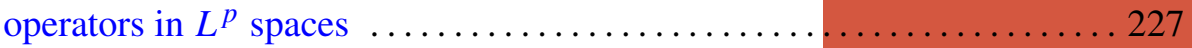

Mark Phillip Thomas, Closed ideals of $l^{1}\left(\omega_{n}\right)$ when $\left\{\omega_{n}\right\}$ is star-shaped $\ldots 237$ 